\title{
Abstract from Current Literatures
}

Title: Growth Failure in Children with Systemic Juvenile Idiopathic Arthritis and Prolonged Inflammation despite Treatment with Biologicals: Late Normalization of Height by Combined Hormonal Therapies.

de Zegher $F^{1}$, Reynaert $N^{1}$, De Somer $L^{2}$, Wouters $\mathrm{C}^{2}$, Roelants $\mathrm{M}^{3}$. Horm Res Paediatr. 2018 Jun 25:17. doi: $10.1159 / 000489778$.

${ }^{1}$ Pediatric Endocrinology, University Hospital Gasthuisberg, Leuven, Belgium. ${ }^{3}$ Pediatric Rheumatology, University Hospital Gasthuisberg, Leuven, Belgium. ${ }^{3}$ Department of Environment and Health, University of Leuven, Leuven, Belgium.

Background: Biologicals targeting the interleukin (IL)-1 or IL-6 pathway are becoming prime choices for the treatment of children with systemic juvenile idiopathic arthritis (sJIA). Up to 1 in 3 sJIA children receiving such treatment continues to have inflammatory activity and to require supraphysiological glucocorticoid doses which may reduce growth velocity for years and may lead to an extremely short stature for age, if not for life. Currently, there is no long-term proposal to normalize the adult height of these children with SJIA.

Methods and Results: We present long-term (up to 10 years), proof-of-concept evidence that the adult stature and adipose body composition of short SJIA children can be normalized with a hormonal combination strategy: (i) pubertal onset is postponed with a gonadotropin-releasing hormone analog (triptorelin) until a minimum height is reached, or until prepubertal growth is exhausted, and (ii) height gain is promoted with growth hormone $\left(\mathrm{H}^{\prime \prime} 50 \mathrm{1} / \mathrm{4g} /\right.$ $\mathrm{kg} /$ day), once inflammation is under control and high glucocorticoid doses are no longer needed. The latter treatment takes advantage of the window of relative glucocorticoid deficiency, which is known to open after prolonged glucocorticoid administration, and to be uniquely favorable to height gain.

Conclusion: A long-term combination of biological and hormonal treatments for short SJIA children can be guided by a simple concept that involves (i) postponement of pubertal development and (ii) growth-promoting therapy after the episodes of major inflammation and high-dose glucocorticoid treatment. Limited long-term experience in short SJIA children suggests that this strategy leads consistently - albeit late - to a normal adult stature.

Infant cortisol stress-response is associated with thymic function and vaccine response.

Huda $\mathrm{MN}^{1,2,3}$, Ahmad $\mathrm{SM}^{3}$, Alam $\mathrm{MJ}^{3}$, Khanam $\mathrm{A}^{3}$, Afsar MNA ${ }^{3}$, Wagatsuma $Y^{4}$, Raqib $R^{3}$, Stephensen $\mathrm{CB}^{1,2}$, Laugero $\mathrm{KD}^{1,2}$. Stress. 2018 Jun 22:1-8. doi: 10.1080/10253890.2018.1484445.

${ }^{1}$ Nutrition Department, University of California , Davis, CA , USA. ${ }^{2}$ US Department of Agriculture , Western Human Nutrition Research Center, Davis, CA , USA. ${ }^{3}$ Enteric and Respiratory Infections Unit, Infectious Diseases Division, icddr,b , Dhaka, Bangladesh. ${ }^{4}$ Department of Clinical Trial and Clinical Epidemiology, Faculty of Medicine, University of Tsukuba, Tsukuba, Japan.

Stress can impair T cell-mediated immunity. To determine if infants with high stress responses had deficits in T-cell mediated immunity, we examined the association of pain-induced cortisol responsiveness with thymic function and vaccine responses in infants. This study was performed among 306 (male $=153$ and female $=153$ ) participants of a randomized, controlled trial examining the effect of neonatal vitamin $A$ supplementation on immune function in Bangladesh (NCT01583972). Salivary cortisol was measured before and 20 min after a needle stick (vaccination) at 6 weeks of age. The thymic index (TI) was determined by ultrasonography at 1, 6, 10 and 15 weeks. T-cell receptor excision circle and blood T-cell concentrations were measured at 6 and 15 weeks. Responses to Bacillus Calmette-Guérin (BCG), tetanus toxoid, hepatitis $B$ virus and oral poliovirus vaccination were assayed at 6 and 15 weeks. Cortisol responsiveness was negatively associated with $\mathrm{Tl}$ at all ages $(\mathrm{p}<.01)$ in boys only, was negatively associated with naïve helper T-cell concentrations in both sexes at both $6(p=.0035)$ 
and 15 weeks ( $p=.0083$ ), and was negatively associated with the delayed-type hypersensitivity (DTH) skin test response to $B C G$ vaccination at 15 weeks $(p=.034)$ in both sexes. Infants with a higher cortisol response to pain have differences in the Tcell compartment and a lower DTH response to vaccination. Sex differences in the immune system were seen as early as 6 weeks of age in these healthy infants.

\section{Comparison of serological assays using pneumococcal proteins or polysaccharides for detection of Streptococcus pneumoniae infection in children with community-acquired pneumonia.}

Borges IC ${ }^{1}$, Andrade $\mathrm{DC}^{2}$, Ekström $\mathrm{N}^{3}$, Virta $\mathrm{C}^{3}$, Melin $\mathrm{M}^{3}$, Saukkoriipi $\mathrm{A}^{4}$, Leinonen $\mathrm{M}^{4}$, Ruuskanen $\mathrm{O}^{5}$, Käyhty $\mathrm{H}^{3}$, Nascimento-Carvalho $\mathrm{CM}^{6}$. J Immunol Methods. 2018 Jun 20. pii: S0022-1759(18)301868. doi: 10.1016/j.jim.2018.06.011.

The aim of this study was to compare the results of serological assays using pneumococcal proteins or polysaccharides for the detection of pneumococcal infection in childhood pneumonia. Serological assays measured IgG against eight pneumococcal proteins (Ply,CbpA,PspA1,PspA2,PcpA,PhtD,StkP-C,PcsB$\mathrm{N})$, C-polysaccharide [in the whole study population, $\mathrm{n} /=/$ 183], or 19 pneumococcal capsular polysaccharides $(1,2,4,5,6 \mathrm{~B}, 7 \mathrm{~F}, 8,9 / \mathrm{V}, 10 \mathrm{~A}, 11 \mathrm{~A}$, $12 \mathrm{~F}, 14,15 \mathrm{~B}, 17 \mathrm{~F}, 18 \mathrm{C}, 19 \mathrm{~F}, 20,23 \mathrm{~F}, 33 \mathrm{~F}$ ) [only in a subgroup of patients, $n /=/ 53$ ] in paired serum samples of children aged $<5 /$ years-old hospitalized with clinical and radiological diagnosis of communityacquired pneumonia. We also performed an inhibition of binding test with the anti-capsular polysaccharide assay in order to confirm the specificity of the antibody responses detected. Invasive pneumococcal pneumonia was investigated by blood culture and PCR (ply-primer). Among 183 children, the antiprotein assay detected antibody response in $77 /$ $183(42.1 \%)$ patients and the anti-C-polysaccharide assay in $28 / 183(15.3 \%$ ) patients. In a subgroup of 53 children, the anti-protein assay detected response in $32 / 53(60.4 \%$ ) patients, the anti-C-polysaccharide assay in $11 / 53(20.8 \%)$ patients, and the anti-capsular polysaccharide in $25 / 53(47.2 \%)$ patients.
Simultaneous antibody responses against e"2 different capsular polysaccharides were detected in $11 / 53(20.8 \%)$ patients and this finding could not be explained by cross-reactivity between different serotypes. Among 13 patients with invasive pneumococcal pneumonia, the sensitivity of the antiprotein assay was $92.3 \%(12 / 13)$, of the anti-Cpolysaccharide assay $30.8 \%(4 / 13)$, and of the anticapsular polysaccharide assay $46.2 \%(6 / 13)$. The serological assay using pneumococcal proteins is more sensitive for the detection of pneumococcal infection in children with pneumonia than the assay using pneumococcal polysaccharides. Future studies on childhood pneumonia aetiology should consider applying serological assays using pneumococcal proteins.

\section{Child abuse and neglect re-report rates for young children with developmental delays.}

Perrigo $\mathrm{JL}^{1}$, Berkovits $\mathrm{LD}^{2}$, Cederbaum JA ${ }^{3}$, Williams $\mathrm{ME}^{4}$, Hurlburt MS ${ }^{5}$. Child Abuse Negl. 2018 Jun 22;83:1-9. doi: 10.1016/j.chiabu.2018.05.029.

The study objective was to examine the likelihood and magnitude of child abuse and neglect (CAN) rereports for young children (0-71 months) with delays in cognitive, language, and adaptive development, compared to typically developing children. The National Survey of Child and Adolescent Well-Being (NSCAW II), a nationally representative and longitudinal survey, was used to examine CAN rereports at two follow-up waves, 18- and 36-months post baseline assessments. Logistic regression models were employed to determine the correlation between number of developmental delays and a CAN re-report at waves 2 and 3 . Results indicate that children with three or more domains of delays had odds 4.73 times higher than children without developmental delays of re-report to CPS at wave 2 but not at wave 3 . In this study, children with multiple developmental delays have elevated rates of CAN re-reports when compared to typically developing children. Allocation of child welfare resources should include strategies for preventing maltreatment risk among children with developmental delays. 